Volume 28 (2020) 43-60

DOI: $10.24330 /$ ieja. 768127

\title{
WEAKLY PRIME AND WEAKLY COMPLETELY PRIME IDEALS OF NONCOMMUTATIVE RINGS
}

\author{
Nico Groenewald \\ Received: 27 April 2019; Revised: 21 June 2020; Accepted: 22 June 2020
}

Communicated by Abdullah Harmancı

\begin{abstract}
Anderson-Smith studied weakly prime ideals for a commutative ring with identity. Hirano, Poon and Tsutsui studied the structure of a ring in which every ideal is weakly prime for rings, not necessarily commutative. In this note we give some more properties of weakly prime ideals in noncommutative rings. We introduce the notion of a weakly prime radical of an ideal. We initiate the study of weakly completely prime ideals and investigate rings for which every proper ideal is weakly completely prime.
\end{abstract}

Mathematics Subject Classification (2020): 16N60

Keywords: Weakly prime ideal, weakly prime radical, weakly completely prime ideal

\section{Definitions and general results}

Anderson-Smith [1] defined a proper ideal $P$ of a commutative ring $R$ with identity to be weakly prime if $0 \neq a b \in P$ implies $a \in P$ or $b \in P$. In [5] Hirano et al. extended the notion of a weakly prime ideal to rings, not necessarily commutative nor with identity. They defined a proper ideal $P$ of the ring to be weakly prime if for ideals $A, B$ of the ring $R, 0 \neq A B \subseteq P$ implies that $A \subseteq P$ or $B \subseteq P$. They studied the structure of rings in which every ideal is weakly prime. Note that by definition, a weakly prime ideal is a proper ideal of a ring. It is therefore not possible that every ideal of a ring is a weakly prime ideal. However, a ring whose zero ideal is prime is called a prime ring. In this sense, every ring is a weakly prime ring since the zero ideal is always weakly prime. We may therefore say that every ideal of a ring is weakly prime when every proper ideal of the ring is a weakly prime ideal. Hirano et al. proved that if every ideal of a ring is weakly prime and $R^{2}=R$, then $\mathcal{P}(R)=N(R)$ and $(\mathcal{P}(R))^{2}=(N(R))^{2}=0$ where $\mathcal{P}(R)$ is the prime radical of $R$ and $N(R)$ the sum of all ideals whose square is zero. They also proved that if every ideal of a right Noetherian ring $R$ with identity is weakly prime then $\mathcal{P}(R)=N(R)=J(R)$ and $(J(R))^{2}=(\mathcal{P}(R))^{2}=(N(R))^{2}=0$, where $J(R)$ is the 
Jacobson radical of $R$. Furthermore, they proved that if every ideal of a ring $R$ is weakly prime then every nonzero ideal of $R / N(R)$ is prime. Motivated by this we further investigate weakly prime ideals in noncommutative rings and also introduce the notion of weakly completely prime ideals.

In this paper, all rings are, as in [5], not necessarily commutative nor with identity. By a ring $R$ with identity, we shall mean that $R$ has a multiplicative identity $1 \neq 0$. By Theorem 3 of Anderson-Smith [1], the following statements are equivalent for an ideal $P$ of a commutative ring $R$ with identity:

(a) $P$ is weakly prime.

(b) For ideals $A$ and $B$ of $R, 0 \neq A B \subseteq P$ implies $A \subseteq P$ or $B \subseteq P$.

For rings that are not necessarily commutative, it is clear that (b) does not imply (a). The standard definition of a prime ideal $P$ for a noncommutative ring $R$ is that for ideals $A$ and $B$ of $R, A B \subseteq P$ implies $A \subseteq P$ or $B \subseteq P$. Equivalent to this is that if $a, b \in R$ such that $a R b \subseteq P$ then $a \in P$ or $b \in P$. From [5] we have the following:

Proposition 1.1. [5, Proposition 2] Let $P$ be an ideal in a ring $R$ with identity. The following statements are equivalent:

(1) $P$ is a weakly prime ideal.

(2) If $a, b \in R$ such that $0 \neq a R b \subseteq P$, then $a \in P$ or $b \in P$.

Let $R$ be a ring. We note that for an element $a \in R,\langle a]=R a+\mathbb{Z} a,[a\rangle=a R+\mathbb{Z} a$ and $\langle a\rangle=\left\{\sum_{i=1}^{n} r_{i} a s_{i}+r a+a s+m a: n \in \mathbb{N}, m \in \mathbb{Z}, r_{i}, s_{i}, r, s \in R\right\}$. Clearly if $R$ is a ring with identity element, then $\langle a]=R a,[a\rangle=a R$ and

$$
\langle a\rangle=\left\{\sum_{i=1}^{n} r_{i} a s_{i}: n \in \mathbb{N}, r_{i}, s_{i} \in R\right\} .
$$

Also, for every two elements $a$ and $b$ of a ring $R$, the following statements are equivalent:

(1) $\langle a\rangle\langle b\rangle=0$.

(2) $a\langle b\rangle=0$.

(3) $\langle a\rangle b=0$.

(4) $a\langle b]=0$.

(5) $[a\rangle b=0$.

The following result is easy to check.

Proposition 1.2. For any ring $R$ and an ideal $P \nsubseteq R$ the following are equivalent:

(1) $P$ is a weakly prime ideal. 
(2) If $a, b \in R$ such that $0 \neq\langle a\rangle\langle b\rangle \subseteq P$, then $a \in P$ or $b \in P$.

(3) If $a, b \in R$ such that $0 \neq\langle a\rangle b \subseteq P$, then $a \in P$ or $b \in P$.

(4) If $a, b \in R$ such that $0 \neq a\langle b\rangle \subseteq P$, then $a \in P$ or $b \in P$.

(5) If $a, b \in R$ such that $0 \neq[a\rangle b \subseteq P$, then $a \in P$ or $b \in P$.

(6) If $a, b \in R$ such that $0 \neq a\langle b] \subseteq P$, then $a \in P$ or $b \in P$.

Analogous to that in [2] we define the concept "twin-zero" for a weakly prime ideal in a noncommutative ring.

Definition 1.3. Let $I$ be a weakly prime ideal of $R$. We say $(a, b)$ is a twin-zero of $I$ if $\langle a\rangle\langle b\rangle=0, a \notin I$ and $b \notin I$.

Proposition 1.4. Let I be a weakly prime ideal of $R$. The following are equivalent:

(1) $(a, b)$ is a twin-zero of $I$ if $\langle a\rangle\langle b\rangle=0, a \notin I$ and $b \notin I$.

(2) $(a, b)$ is a twin-zero of $I$ if $\langle a\rangle b=0, a \notin I$ and $b \notin I$.

(3) $(a, b)$ is a twin-zero of $I$ if $a\langle b\rangle=0, a \notin I$ and $b \notin I$.

Note that if $I$ is a weakly prime ideal of $R$ that is not a prime ideal then $I$ has a twin-zero $(a, b)$ for some $a, b \in R$.

Lemma 1.5. Let $I$ be a weakly prime ideal of $R$ and suppose that $(a, b)$ is a twinzero of $I$ for some $a, b \in R$. Then $\langle a\rangle I=I\langle b\rangle=0$.

Proof. Suppose that $\langle a\rangle I \neq 0$. Then there exists $i \in I$ such that $\langle a\rangle i \neq 0$. Hence $0 \neq\langle a\rangle(b+i)=\langle a\rangle b+\langle a\rangle i=\langle a\rangle i \subseteq I$, since $(a, b)$ is a twin-zero of $I$. Because $a \notin I$ and $I$ weakly prime, we have $b+i \in I$, and hence $b \in I$, a contradiction. Thus $\langle a\rangle I=0$. Now, suppose $I\langle b\rangle \neq 0$. Then there exists $t \in I$ such that $t\langle b\rangle \neq 0$. Hence $0 \neq(a+t)\langle b\rangle \subseteq I$. Since $b \notin I$ and $I$ weakly prime, we have $a+t \in I$, and hence $a \in I$, a contradiction. Thus $I\langle b\rangle=0$.

Theorem 1.6. [5, Proposition 1] If $P$ is weakly prime but not prime then $P^{2}=0$.

Proof. Let $(a, b)$ be a twin-zero of $P$. Suppose that $P^{2} \neq 0$. Then by Lemma 1.5 , we have $0 \neq(\langle a\rangle+P)(\langle b\rangle+P)=\langle a\rangle\langle b\rangle+\langle a\rangle P+P\langle b\rangle+P^{2} \subseteq P$. Thus $(\langle a\rangle+P) \subseteq P$ or $(\langle b\rangle+P) \subseteq P$ and hence $a \in P$ or $b \in P$ a contradiction since $(a, b)$ is a twin-zero of $P$. Therefore $P^{2}=0$.

Corollary 1.7. Let $R$ be a ring and let $P$ an ideal of $R$. If $P^{2} \neq 0$ then $P$ is prime if and only if $P$ is weakly prime.

Proof. This follows from Theorem 1.6. 
Corollary 1.8. Let $\mathcal{P}(R)$ denote the prime radical of the ring $R$, i.e. the intersection of all the prime ideals of $R$. If $P$ is a weakly prime ideal which is not a prime ideal, then $P \subseteq \mathcal{P}(R)$.

Proof. This follows since $\mathcal{P}(R)$ is a semi-prime ideal of $R$ and from Theorem 1.6 $P^{2}=0 \subseteq \mathcal{P}(R)$.

Corollary 1.9. Let $P$ be a weakly prime ideal of $R$. Then

(i) Either $P \subseteq \mathcal{P}(R)$ or $\mathcal{P}(R) \subseteq P$.

(ii) If $P \subset \mathcal{P}(R)$, then $P$ is not prime.

(iii) If $\mathcal{P}(R) \subset P$, then $P$ is prime.

(iv) If $P=\mathcal{P}(R)$, then $P$ may or may not be prime.

Hence, if $R$ is a prime ring then $P$ is weakly prime if and only if $P=0$ or $P$ is prime.

It should be noted that a proper ideal $P$ with property that $P^{2}=\{0\}$ need not be weakly prime. Take $R=\left[\begin{array}{ll}\mathbb{Q} & \mathbb{R} \\ 0 & \mathbb{Q}\end{array}\right]$ and $P=\left[\begin{array}{ll}0 & \mathbb{R} \\ 0 & 0\end{array}\right]$. Clearly $P^{2}=0$ yet $P$ is not weakly prime since $\left[\begin{array}{ll}0 & 0 \\ 0 & 0\end{array}\right] \neq\left[\begin{array}{ll}3 & 0 \\ 0 & 0\end{array}\right]\left[\begin{array}{ll}\mathbb{Q} & \mathbb{R} \\ 0 & \mathbb{Q}\end{array}\right]\left[\begin{array}{ll}0 & 2 \\ 0 & 3\end{array}\right] \subseteq P$.

In [4] Birkenmeier et al. introduced the notion of a 2-primal ideal and a 2-primal ring.

Definition 1.10. [4, Definition 2.1] Let $R$ be a ring and $I$ an ideal of $R$. The ring $R$ is 2-primal if the prime radical $\mathcal{P}(R)$ of $R$ is equal to the set of nilpotent elements of $R$. The ideal $I$ is 2-primal if the factor ring $R / I$ is a 2-primal ring.

Proposition 1.11. If $P$ is a weakly prime ideal that is not a prime ideal of the ring $R$, then $R$ is 2-primal if and only if $P$ is a 2-primal ideal.

Proof. This follows from Corollary 1.8 and [4, Proposition 2.4].

Lemma 1.12. Let $I$ be a weakly prime ideal of $R$ and suppose that $(a, b)$ is a twin-zero of I. If $\langle a\rangle r \subseteq I$ for some $r \in R$, then $\langle a\rangle r=0$.

Proof. Suppose that $0 \neq\langle a\rangle r \subseteq I$ for some $r \in R$. Then $r \in I$ since $I$ is weakly prime and $(a, b)$ is a twin-zero of $I$. Now, since $\langle a\rangle r \subseteq\langle a\rangle I$, we have that $\langle a\rangle r=0$ from Lemma 1.5, a contradiction.

Theorem 1.13. Let $I$ be a weakly prime ideal of $R$ and suppose that $A B \subseteq I$ for some ideals $A, B$ of $R$. If I has a twin-zero $(a, b)$ for some $a \in A$ and $b \in B$, then $A B=0$. 
Proof. Suppose that $I$ has a twin-zero $(a, b)$ for some $a \in A$ and $b \in B$ and assume that $c d \neq 0$ for some $c \in A$ and $d \in B$. Since $0 \neq c d \in\langle c\rangle d \subseteq A B \subseteq I$ and $I$ weakly prime, we have $c \in I$ or $d \in I$. Without loss of generality, we may assume that $c \in I$. Since $I^{2}=0$ by Theorem 1.6 and $0 \neq c d \in I$, we conclude that $d \notin I$. Since $\langle a\rangle d \subseteq A B \subseteq I$ it follows from Lemma 1.12 that $\langle a\rangle d=0$. Hence $a\langle d\rangle=0$. Now, $(a+c)\langle d\rangle=a\langle d\rangle+c\langle d\rangle=c\langle d\rangle \subseteq A B \subseteq I$. Since $c d \neq 0, d \notin I$ and $I$ weakly prime, we have $(a+c) \in I$. Hence $a \in I$, a contradiction. Thus $A B=0$.

Theorem 1.14. For a proper ideal $P$ of $R$ the following statements are equivalent:

(1) $P$ is weakly prime.

(2) For $x \in R-P,(P:\langle x])=\{p \in R: p\langle x] \subseteq P\}=P \cup(0:\langle x])$.

(3) For $x \in R-P,(P:\langle x])=P$ or $(P:\langle x])=(0:\langle x])$.

Proof. $(1) \Rightarrow(2)$ Let $y \in(P:\langle x])$ where $x \in R-P$. Now $y\langle x] \subseteq P$. If $y\langle x] \neq 0$ then $P$ weakly prime gives $y \in P$. If $y\langle x]=0$, then $y \in(0:\langle x])$. So $(P:\langle x]) \subseteq$ $P \cup(0:\langle x])$. As the reverse containment holds for any ideal $P$, we have equality.

$(2) \Rightarrow(3)$ Suppose $(P:\langle x])=P \cup(0:\langle x])$ where $x \in R-P$. Since $P$ and $(0:\langle x])$ are both ideals, we have $(P:\langle x])=P$ or $(P:\langle x])=(0:\langle x])$.

$(3) \Rightarrow(1)$ Let $x, y \in R$ such that $0 \neq y\langle x] \subseteq P$. If $x \in P$, then we are done. So suppose $x \in R-P$, then $(P:\langle x]) \neq(0:\langle x])$ and from $(3)$, we have $(P:\langle x])=P$. Hence $y \in P$ and we are done.

Lemma 1.15. Let $R$ be a ring and $P$ an ideal of $R$. Then the following are equivalent.

(1) $P$ is weakly prime.

(2) For any ideals $I, J$ of $R$ with $P \subset I$ and $P \subset J$, we have either $I J=0$ or $I J \nsubseteq P$.

(3) For any ideals $I, J$ of $R$ with $I \nsubseteq P$ and $J \nsubseteq P$, we have either $I J=0$ or $I J \nsubseteq P$.

Proof. $(1) \Rightarrow(2)$ and (3) $\Rightarrow(1)$ are clear.

(2) $\Rightarrow(3)$ Let $I, J$ be ideals of $R$ with $I \nsubseteq P$ and $J \nsubseteq P$. If $I J=0$, then we are done, so suppose that $I J \neq 0$. Let $i \in I$ and $j \in J$ such that $0 \neq i j$. Also, since $I \nsubseteq P$ and $J \nsubseteq P$ there exist $i_{1} \in I$ and $j_{1} \in J$ such that $i_{1}, j_{1} \notin P$. Now $P \subset\left\langle i_{1}\right\rangle+\langle i\rangle+P$ and $P \subset\left\langle j_{1}\right\rangle+\langle j\rangle+P$. Furthermore, $0 \neq i j \in\langle i\rangle\langle j\rangle \subseteq$ $\left(\left\langle i_{1}\right\rangle+\langle i\rangle+P\right)\left(\left\langle j_{1}\right\rangle+\langle j\rangle+P\right)$. Hence from our assumption we have $\left(\left\langle i_{1}\right\rangle+\langle i\rangle+\right.$ $P)\left(\left\langle j_{1}\right\rangle+\langle j\rangle+P\right) \nsubseteq P$ and it follows that $P+\left\langle i_{1}\right\rangle\left(\left\langle j_{1}\right\rangle+\langle j\rangle\right)+\langle i\rangle\left(\left\langle j_{1}\right\rangle+\langle j\rangle\right) \nsubseteq P$.

For this to be true we must have $I J \nsubseteq P$. 
Proposition 1.16. Any weakly prime ideal $P$ in a ring $R$ contains a minimal weakly prime ideal.

Proof. Apply Zorn's Lemma to the family of weakly prime ideals of $R$ contained in $P$. It suffices to check that, for any chain of weakly prime ideals $\left\{P_{i}: i \in I\right\}$ in $P$, the intersection $P^{\prime}=\cap P_{i}$ is weakly prime. Let $A$ and $B$ be ideals of $R$ such that $0 \neq A B \subseteq P^{\prime}$. Suppose that $A \nsubseteq P^{\prime}$ and $B \nsubseteq P^{\prime}$. Then there exist $a \in A \backslash P^{\prime}$ and $b \in B \backslash P^{\prime}$ and we have $a \notin P_{i}$ and $b \notin P_{j}$ for some $i, j \in I$. If, say $P_{i} \subseteq P_{j}$, then both $a, b$ are outside $P_{i}$. Since $P_{i}$ is weakly prime we have $\langle a\rangle\langle b\rangle=0$ or $\langle a\rangle\langle b\rangle \nsubseteq P_{i}$. Because $\langle a\rangle\langle b\rangle \subseteq A B \subseteq P^{\prime} \subseteq P_{i}$ we must have $\langle a\rangle\langle b\rangle=0$. Hence $(a, b)$ is a twin zero for $P_{i}$. It now follows from Theorem 1.13 that $A B=0$. This contradicts our assumption hence $A \subseteq P^{\prime}$ or $B \subseteq P^{\prime}$ and therefore $P^{\prime}$ is a weakly prime ideal.

Proposition 1.17. Let $R$ be a Noetherian ring and $I \neq R$ an ideal. The set of minimal weakly prime ideals containing I is finite.

Proof. Assume the result is false and choose $I \neq R$ an ideal maximal with respect to the property that $I \neq R$ and that there are infinitely many weakly prime ideals containing $I$. This is possible as $R$ is Noetherian. Then clearly $I$ is not a weakly prime ideal so there exist elements $a, b \in R$ such that $0 \neq\langle a\rangle\langle b\rangle \subseteq I$ but $a \notin I$ and $b \notin I$. Let $J=I+\langle a\rangle$ and $K=I+\langle b\rangle$. Now $J$ and $K$ properly contain $I$. Furthermore, $0 \neq\langle a\rangle\langle b\rangle \subseteq J K=(I+\langle a\rangle)(I+\langle b\rangle) \subseteq I$. Since $I$ is weakly prime we must have $J \subseteq I$ or $K \subseteq I$. Note that any weakly prime ideal containing $I$ must contain either $J$ or $K$. In particular, any weakly prime ideal minimal over $I$ is minimal over either $J$ or $K$. But $J$ and $K$ each have only finitely many minimal weakly primes (by choice of $I$ ), a contradiction.

Theorem 1.18. Let $R$ be a decomposable ring with identity. If $P$ is a weakly prime ideal of $R$, then either $P=0$ or $P$ is prime.

Proof. Suppose that $R=R_{1} \times R_{2}$. Let $P=P_{1} \times P_{2}$ be a weakly prime ideal of $R$. We can assume that $P \neq 0$. Now, let $A$ be a non-zero ideal of $R_{1}$ and $B$ be a nonzero ideal of $R_{2}$ such that $0 \neq A \times B \subseteq P$. Then $0 \neq\left(A \times R_{2}\right)\left(R_{1} \times B\right) \subseteq A \times B \subseteq P$ which implies $A \times R_{2} \subseteq P$ or $R_{1} \times B \subseteq P$. Suppose that $A \times R_{2} \subseteq P$. Then $0 \times R_{2} \subseteq P$ and so $P=P_{1} \times R_{2}$. We show that $P_{1}$ is a prime ideal of $R_{1}$. Let $A_{1}$ and $B_{1}$ be ideals of $R_{1}$ such that $A_{1} B_{1} \subseteq P_{1}$. Then $(0,0) \neq\left(A_{1} \times R_{2}\right)\left(B_{1} \times R_{2}\right) \subseteq$ $A_{1} B_{1} \times R_{2} \subseteq P$, so $A_{1} \times R_{2} \subseteq P$ or $B_{1} \times R_{2} \subseteq P$ and hence $A_{1} \subseteq P_{1}$ or $B_{1} \subseteq P_{1}$. So $P$ is a prime ideal of $R$. The case where $\left(R_{1} \times B\right) \subseteq P$ is similar. 
Proposition 1.19. Let $A \subseteq P$ be a proper ideal of a ring $R$. Then the following holds:

(1) If $P$ is weakly prime, then $P / A$ is weakly prime.

(2) If $A$ and $P / A$ are weakly prime, then $P$ is weakly prime.

Proof. (1) Let $0 \neq((\langle a\rangle+A)(b+A)) / A=(\langle a\rangle b+A) / A \subseteq P / A$ where $a, b \in R$, so $\langle a\rangle b \subseteq P$. If $\langle a\rangle b=0 \subseteq A$, then $((\langle a\rangle+A)(b+A)) / A=0$ a contradiction. Hence $\langle a\rangle b \neq 0$ and since $\langle a\rangle b \subseteq P$ and $P$ weakly prime, we get $a \in P$ or $b \in P$. Hence $(a+A) \in P / A$ or $(b+A) \in P / A$ as required.

(2) Let $0 \neq\langle a\rangle b \subseteq P$ where $a, b \in R$ so that $((\langle a\rangle+A)(b+A)) / A \subseteq P / A$. If $\langle a\rangle b \subseteq A$, then since $A$ is weakly prime, we get $a \in A \subseteq P$ or $b \in A \subseteq P$. If $\langle a\rangle b \nsubseteq A$, then $0 \neq((\langle a\rangle+A)(b+a)) / A \subseteq P / A$. Now, since $P / A$ is weakly prime, we get $(a+A) \in P / A$ or $(b+A) \in P / A$. Hence $a \in P$ or $b \in P$ as needed.

Theorem 1.20. Let $P$ and $Q$ be weakly prime ideals of a ring $R$ that are not prime. Then $P+Q$ is a weakly prime ideal of $R$.

Proof. Since $(P+Q) / Q \cong Q /(P \cap Q)$ we get that $(P+Q) / Q$ is weakly prime by Proposition 1.19 (1). Now the assertion follows from Proposition 1.19 (2).

\section{Idealization}

We now show how to construct weakly prime ideals using the Method of Idealization. In what follows, $R$ is a ring (associative, not necessarily commutative and not necessarily with identity) and $M$ is an $R$ - $R$-bimodule. The idealization of $M$ is the ring $R \boxplus M$ with $(R \boxplus M,+)=(R,+) \oplus(M,+)$ and the multiplication is given by $(r, m)(s, n)=(r s, r n+m s) . R \boxplus M$ itself is, in a canonical way, an $R$ - $R$-bimodule and $M \simeq 0 \boxplus M$ is a nilpotent ideal of $R \boxplus M$ of index 2. We also have $R \simeq R \boxplus 0$ and the latter is a subring of $R \boxplus M$. Note also that $R \boxplus M$ is a subring of the Morita ring $\left[\begin{array}{cc}R & M \\ 0 & R\end{array}\right]$ via the mapping $(r, m) \mapsto\left[\begin{array}{cc}r & m \\ 0 & r\end{array}\right]$. We will require some knowledge about the ideal structure of $R \boxplus M$. If $I$ is an ideal of $R$ and $N$ is an $R$-R-bi-submodule of $M$, then $I \boxplus N$ is an ideal of $R \boxplus M$ if and only if $I M+M I \subseteq N$. It follows from [7] that the prime ideals of $R \boxplus M$ are exactly the ideals of the form $I \boxplus M$ where $I$ is a prime ideal of $R$.

If $R$ is a ring with identity then $(a, b)$ is a twin zero of an ideal $I$ of $R$ if $a R b=0$ and $a \notin I$ and $b \notin I$. 
Theorem 2.1. Let $R$ be a ring with identity and $M$ an $R$-R-bimodule, with $I$ a proper ideal of $R$. Then $I \boxplus M$ is a weakly prime ideal of $R \boxplus M$ if and only if $I$ is a weakly prime ideal of $R$ and for any twin zero $(a, b)$ of $I$ we have $a M=M b=0$.

Proof. Suppose $I \boxplus M$ is a weakly prime ideal of $R \boxplus M$. Let $0 \neq a R b \subseteq I$ where $a, b \in R$. Now $(0,0) \neq(a, 0) R \boxplus M(b, 0) \subseteq I \boxplus M$ and $I \boxplus M$ a weakly prime ideal gives $(a, 0) \in I \boxplus M$ or $(b, 0) \in I \boxplus M$. Hence $a \in I$ or $b \in I$. So $I$ is weakly prime. Now suppose $(a, b)$ is a twin zero of $I$. We claim that $a M=M b=0$. Assume say $a M \neq 0$, so there exists $m \in M$ such that $a m \neq 0$. Now we have $(0,0) \neq(a, 0)(1,0)(b, m) \in(a, 0) R \boxplus M(b, m) \subseteq a R b \boxplus M=0 \boxplus M \subseteq I \boxplus M$. But $(a, 0) \notin I \boxplus M$ and $(b, m) \notin I \boxplus M$ contradicting the fact that $I \boxplus M$ is a weakly prime ideal.

Conversely, assume $(0,0) \neq(a, m) R \boxplus M(b, n) \subseteq I \boxplus M$ for $a, b \in R$ and $n, m \in$ $M$. We have $a R b \subseteq I$. Two cases are possible:

Case 1: $0 \neq a R b \subseteq I$. Now $I$ a weakly prime ideal of $R$ gives $a \in I$ or $b \in I$. Hence $(a, m) \in I \boxplus M$ or $(b, n) \in I \boxplus M$ as desired.

Case 2: $0=a R b \subseteq I$. We may assume $a \notin I$ and $b \notin I$. Hence $(a, b)$ is a twin zero of $I$ and from assumption $a M=M b=0$. Now $(a, m) R \boxplus M(b, n) \subseteq$ $(a R b, a M+a M b+M b)=(0,0)$ a contradiction.

Corollary 2.2. Let $R$ be a semi-prime ring with identity which is not a prime ring and $M$ be an $R$-R-bimodule. Then the unique weakly prime ideal which is not a prime ideal of $R \boxplus M$ which has the form $I \boxplus M$ where $I$ is an ideal of $R$, is the ideal $0 \boxplus M$.

Proof. Let $I$ be an ideal of $R$ and $J:=I \boxplus M$ such that $J$ is a weakly prime ideal which is not a prime ideal of $R$. Then $I$ is a weakly prime ideal of $R$ which is not a prime ideal of $R$ (recall that $I \boxplus M$ is prime if and only if $I$ is prime). From Corollary $1.8 I \subseteq \mathcal{P}(R)=0$. This means that $J=0 \boxplus M$. The zero ideal $\{0\}$ is a weakly prime ideal of $R$. Let $(a, b)$ be a twin zero of $\{0\}$. Hence $a R b=0$ with $a \neq 0$ and $b \neq 0$. We claim that $a M=M b=0$. Without loss of generality, we may assume $a M \neq 0$. Then, there exists $n \in M$ such that an $\neq 0$. Now, $(0,0) \neq(0, a n)=(a, 0)(1,0)(b, n) \in(a, 0) R \boxplus M(b, n) \subseteq a R b \boxplus M=0 \boxplus M=J$ and neither $(a, 0) \in J$ nor $(b, n) \in J$, a desired contradiction since $J$ is a weakly prime ideal of $R \boxplus M$. On the other hand, by Theorem 1.6 and since $J^{2}=0, J$ is not a prime ideal of $R \boxplus M$, which completes the proof. 


\section{The weakly prime radical}

Motivated by the work of Beiranvand et al. in [3] we introduce the notion of a weakly prime radical of an ideal of a ring.

We begin this section with the definition of weakly m-systems.

Definition 3.1. Let $R$ be a ring. A nonempty set $S \subseteq R \backslash\{0\}$ is called a weakly m-system if, for ideals $A$ and $B$ of $R$ if $A \cap S \neq \emptyset, B \cap S \neq \emptyset$ and $A B \neq 0$ then $A B \cap S \neq \emptyset$.

Lemma 3.2. For a proper ideal $P$ of $R$ let $S=R \backslash P$. Then $P$ is a weakly prime ideal of $R$ if and only if $S$ is a weakly m-system.

Proof. Suppose $S=R \backslash P$. Let $A$ and $B$ be ideals in $R$ such that $A \cap S \neq \emptyset$, $B \cap S \neq \emptyset$ and $A B \neq 0$. If $A B \cap S=\emptyset$ then $A B \subseteq P$. Since $P$ is weakly prime, and $A B \neq 0, A \subseteq P$ or $B \subseteq P$. It follows that $A \cap S=\emptyset$ or $B \cap S=\emptyset$, a contradiction. Therefore, $S$ is a weakly m-system in $R$. Conversely, let $S=R \backslash P$ be a weakly m-system in $R$. Suppose $A B \subseteq P$ and $A B \neq 0$, where $A$ and $B$ are ideals of $R$. If $A \nsubseteq P$ and $B \nsubseteq P$, then $A \cap S \neq \emptyset$ and $B \cap S \neq \emptyset$. Since $S$ is a weakly m-system $A B \cap S \neq \emptyset$, a contradiction. Therefore, $P$ is a weakly prime ideal of $R$.

The following proposition offers several characterizations of a weakly m-system $S$ when it is the complement of an ideal.

Proposition 3.3. Let $R$ be a ring and $P$ be a proper ideal of $R$ and let $S:=R \backslash P$. Then the following statements are equivalent:

(1) $P$ is weakly prime.

(2) $S$ is a weakly m-system.

(3) for left ideals $A, B \subseteq R$, if $A \cap S \neq \emptyset, B \cap S \neq \emptyset$ and $A B \neq 0$ then $A B \cap S \neq \emptyset$.

(4) for right ideals $A, B \subseteq R$ if $A \cap S \neq \emptyset, B \cap S \neq \emptyset$ and $A B \neq 0$, then $A B \cap S \neq \emptyset$.

(5) for each $a, b \in R$, if $a, b \in S$ and $\langle a\rangle\langle b\rangle \neq 0$, then $\langle a\rangle\langle b\rangle \cap S \neq \emptyset$.

Proof. $(1) \Leftrightarrow(2)$ follows from Lemma 3.2.

$(2) \Rightarrow(3) \Rightarrow(4) \Rightarrow(5)$ and $(5) \Rightarrow(1)$ follows from Proposition 1.2 and [5, Proposition 2].

Proposition 3.4. Let $S \subseteq R$ be a weakly m-system, and let $P$ an ideal of $R$ maximal with respect to the property that $P$ is disjoint from $S$. Then $P$ is a weakly prime ideal. 
Proof. Suppose $0 \neq A B \subseteq P$, where $A$ and $B$ are ideals of $R$. If $A \nsubseteq P$ and $B \nsubseteq P$, then by the maximal property of $P$, we have, $(P+A) \cap S \neq \emptyset$ and $(P+B) \cap S \neq \emptyset$. Furthermore, $0 \neq A B \subseteq(P+A)(P+B) \subseteq P$. Thus, since $S$ is a weakly m-system $(P+A)(P+B) \cap S \neq \emptyset$ and it follows that $(P+A)(P+B) \nsubseteq P$. For this to happen, we must have $A B \nsubseteq P$, a contradiction. Thus, $P$ must be a weakly prime ideal.

Next we need a generalization of the notion of $\sqrt{ } A$ for any ideal of $A$ of $R$. We adopt the following:

Definition 3.5. Let $R$ be a ring. For an ideal $A$ of $R$, if there is a weakly prime ideal containing $A$, then we define $\mathcal{P}_{w}(A):=\{a \in R$ : every weakly m-system containing $a$ meets $A\}$. If there is no weakly prime ideal containing $A$, then we put $\mathcal{P}_{w}(A)=R$.

For an ideal $A$ of $R$, observe that $A$ and $\mathcal{P}_{w}(A)$ are contained in precisely the same weakly prime ideals of $R$.

Theorem 3.6. Let $A$ be an ideal of the ring $R$. Then either $\mathcal{P}_{w}(A)=R$ or $\mathcal{P}_{w}(A)$ equals the intersection of all the weakly prime ideals of $R$ containing $A$.

Proof. Suppose that $\mathcal{P}_{w}(A) \neq R$. This means that $\{P: P$ is a weakly prime ideal of $R$ and $A \subseteq P\} \neq \emptyset$. We first prove that $\mathcal{P}_{w}(A) \subseteq\{P: P$ is a weakly prime ideal of $R$ and $A \subseteq P\}$. Let $m \in \mathcal{P}_{w}(A)$ and $P$ be any weakly prime ideal of $R$ containing $A$. Consider the weakly m-system $R \backslash P$. This weakly m-system cannot contain $m$, for otherwise it meets $A$ and hence also $P$. Therefore, we have $m \in P$. Conversely, assume $m \notin \mathcal{P}_{w}(A)$. Then, by Definition 3.5, there exists a weakly m-system $S$ containing $m$ which is disjoint from $A$. By Zorn's Lemma, there exists an ideal $P \supseteq A$ which is maximal with respect to being disjoint from $S$. By Proposition 3.4, $P$ is a weakly prime ideal of $R$ and we have $m \notin P$, as desired.

Theorem 3.7. Let $A$ be an ideal of the ring $R$. Then $\mathcal{P}_{w}(A)$ equals the intersection of all the weakly minimal weakly prime ideals of $R$ containing $A$.

Proof. This follows from Theorem 3.6 and Proposition 1.16.

Example 3.8. Let $R=\left\{\left[\begin{array}{ll}a & b \\ 0 & 0\end{array}\right]: a, b \in \mathbb{Z}_{4}, b \in\{0,2\}\right\} . R$ has 2 proper ideals $P_{1}=\left\{\left[\begin{array}{ll}0 & 0 \\ 0 & 0\end{array}\right],\left[\begin{array}{ll}0 & 2 \\ 0 & 0\end{array}\right]\right\}$ and $P_{2}=\left\{\left[\begin{array}{ll}0 & 0 \\ 0 & 0\end{array}\right],\left[\begin{array}{ll}2 & 0 \\ 0 & 0\end{array}\right]\right\} . P_{1}$ is a weakly 
prime ideal which is not a prime ideal since

$$
\left\{\left[\begin{array}{ll}
0 & 0 \\
0 & 0
\end{array}\right],\left[\begin{array}{ll}
2 & 0 \\
0 & 0
\end{array}\right]\right\}\left\{\left[\begin{array}{ll}
0 & 0 \\
0 & 0
\end{array}\right],\left[\begin{array}{ll}
2 & 0 \\
0 & 0
\end{array}\right]\right\}=\left\{\left[\begin{array}{ll}
0 & 0 \\
0 & 0
\end{array}\right]\right\} \subseteq P_{1}
$$

but $\left\{\left[\begin{array}{ll}0 & 0 \\ 0 & 0\end{array}\right],\left[\begin{array}{ll}2 & 0 \\ 0 & 0\end{array}\right]\right\} \nsubseteq P_{1} . \quad \mathcal{P}_{w}\left(P_{1}\right)=\left\{\left[\begin{array}{ll}0 & 0 \\ 0 & 0\end{array}\right],\left[\begin{array}{ll}0 & 2 \\ 0 & 0\end{array}\right]\right\}$ and $\mathcal{P}_{w}\left(P_{2}\right)=R$.

Example 3.9. [5, Example 5] Let $R$ be the noncommutative ring of endomorphisms of a countably infinite dimensional vector space. $R$ is a prime ring with exactly one nonzero proper ideal $P$. Every ideal of $S_{1}=R \boxplus P$ is weakly prime: the maximum ideal $P_{1}=P \boxplus P$ is idempotent and the nonzero minimal ideal $P_{2}=0 \boxplus P$ is nilpotent, both of which are prime. Let $S_{2}=S_{1} \boxplus P_{2}$. Every ideal of $S_{2}$ is weakly prime: The maximum ideal $Q_{1}=P_{1} \boxplus P_{2}$ is idempotent and the three nonzero nilpotent ideals are $Q_{2}=P_{2} \boxplus P_{2}, Q_{3}=0 \boxplus P_{2}$, and $Q_{4}=P_{2} \boxplus 0 . \quad Q_{3}$ and $Q_{4}$ are not prime ideals since $0=Q_{2}^{2} \subseteq Q_{3}$ and $0=Q_{2}^{2} \subseteq Q_{4}$. For the weakly prime and prime radicals of the ideal $Q_{3}$ we have $\mathcal{P}_{w}\left(Q_{3}\right)=Q_{3} \cap Q_{2} \cap Q_{1}=Q_{3}$ and $\mathcal{P}\left(Q_{3}\right)=Q_{2} \cap Q_{1}=Q_{2}$.

\section{Weakly completely prime ideals}

Recall that an ideal $P$ of the ring $R$ is a completely prime ideal if $a b \in P$ implies $a \in P$ or $b \in P$ for $a, b \in R$.

Definition 4.1. A proper ideal $I$ of a ring $R$ is weakly completely prime if $0 \neq$ $a b \in P$ implies $a \in P$ or $b \in P$ for $a, b \in R$.

Analogous to that in [2] we define the concept "c-twin-zero" for a weakly completely prime ideal in a noncommutative ring.

Definition 4.2. Let $I$ be a weakly completely prime ideal of $R$. We say $(a, b)$ is a c-twin-zero of $I$ if $a b=0, a \notin I$ and $b \notin I$.

Note that if $I$ is a weakly completely prime ideal of $R$ that is not a completely prime ideal, then $I$ has a c-twin-zero $(a, b)$ for some $a, b \in R$.

Lemma 4.3. Let $I$ be a weakly completely prime ideal of $R$ and suppose that $(a, b)$ is a c-twin-zero of $I$ for some $a, b \in R$. Then $a I=I b=0$.

Proof. Same as the proof of [2, Theorem 3.2].

Lemma 4.4. Let I be a weakly completely prime ideal of $R$ and suppose that $(a, b)$ is a c-twin-zero of $I$. If ar $\in I$ for some $r \in R$, then ar $=0$. 
Proof. Suppose that $0 \neq a r \in I$ for some $r \in R$. Then $r \in I$ since $I$ is weakly completely prime and $(a, b)$ is a c-twin-zero of $I$. Now, since $a r \in a I$, we have that $a r=0$ from Lemma 4.3, a contradiction.

Theorem 4.5. If $P$ is weakly completely prime but not completely prime, then $P^{2}=0$.

Proof. Let $(a, b)$ be a c-twin-zero of $P$. Suppose that $p_{1} p_{2} \neq 0$ for some $p_{1}, p_{2} \in P$. Then by Lemma 4.3 , we have $0 \neq\left(a+p_{1}\right)\left(b+p_{2}\right) \in P$. Thus $\left(a+p_{1}\right) \in P$ or $\left(b+p_{2}\right) \in P$ and hence $a \in P$ or $b \in P$ a contradiction since $(a, b)$ is a c-twin-zero of $P$. Therefore $P^{2}=0$.

Corollary 4.6. Let $R$ be a ring and let $P$ an ideal of $R$. If $P^{2} \neq 0$ then $P$ is completely prime if and only if $P$ is weakly completely prime.

Proof. This follows from Theorem 4.5.

Remark 4.7. Let $\mathcal{N}_{g}(R)$ denote the generalized nil (completely prime) radical of the ring $R$, i.e. the intersection of all the completely prime ideals of $R$. If $P$ is a weakly completely prime ideal which is not a completely prime ideal, then $P \subseteq \mathcal{N}_{g}(R)$. This follows since $\mathcal{N}_{g}(R)$ is a semi-prime ideal of $R$ and from Theorem $4.5, P^{2}=0 \subseteq \mathcal{N}_{g}(R)$.

Corollary 4.8. Let $P$ be a weakly completely prime ideal of $R$. Then

(i) Either $P \subseteq \mathcal{N}_{g}(R)$ or $\mathcal{N}_{g}(R) \subseteq P$.

(ii) If $P \subset \mathcal{N}_{g}(R)$, then $P$ is not completely prime.

(iii) If $\mathcal{N}_{g}(R) \subset P$, then $P$ is completely prime.

(iv) If $P=\mathcal{N}_{g}(R)$, then $P$ may or may not be completely prime.

Hence, if $R$ is a reduced ring then $P$ is weakly completely prime if and only if $P=0$ or $P$ is completely prime.

It should be noted that a proper ideal $P$ with property that $P^{2}=\{0\}$ need not be weakly completely prime. Take $R=\left[\begin{array}{ll}\mathbb{Q} & \mathbb{R} \\ 0 & \mathbb{Q}\end{array}\right]$ and $P=\left[\begin{array}{ll}0 & \mathbb{R} \\ 0 & 0\end{array}\right]$. Clearly $P^{2}=$ 0 , yet $P$ is not weakly completely prime since $\left[\begin{array}{ll}0 & 0 \\ 0 & 0\end{array}\right] \neq\left[\begin{array}{ll}3 & 0 \\ 0 & 0\end{array}\right]\left[\begin{array}{ll}0 & 2 \\ 0 & 3\end{array}\right]=$ $\left[\begin{array}{ll}0 & 6 \\ 0 & 0\end{array}\right] \in P$.

Proposition 4.9. Let $P$ be a weakly completely prime ideal of the ring $R$. If $a \in R$ and $B$ a subset of $R$ such that $0 \neq a B \subseteq P$, then $a \in P$ or $B \subseteq P$. 
Proof. Suppose $a \in R$ and $B$ a subset of $R$ such that $0 \neq a B \subseteq P$. If $a \in P$, then we are done. So, suppose $a \notin P$. For every $b \in B$ such that $0 \neq a b \in P$, we have $b \in P$ since $P$ is weakly completely prime. If $t \in B$ such that $0=a t \in P$ and $t \notin P$, then $(a, t)$ is a c-twin-zero of $P$. Because $a B \subseteq P$, it follows from Lemma 4.4 that $a B=0$, a contradiction and therefore $t \in P$ and we have $B \subseteq P$.

Theorem 4.10. Let $P$ be a weakly completely prime ideal of $R$, then $P$ is a weakly prime ideal of $R$.

Proof. Let $P$ be a weakly completely prime ideal of $R$ and suppose that $0 \neq a\langle b\rangle \subseteq$ $P$ for $a, b \in R$. If $a \in P$, then we are done, so suppose $a \notin P$. Now, since $P$ is weakly completely prime it follows from Proposition 4.9 that $b \in\langle b\rangle \subseteq P$ and we are done.

Example 4.11. Not every weakly prime ideal is a weakly completely prime ideal. Let $M_{2}(\mathbb{Z})$ be the full matrix ring with entries from the ring of integers $\mathbb{Z} . M_{2}(2 \mathbb{Z})$ is a prime ideal and hence also weakly prime ideal of $M_{2}(\mathbb{Z})$. To show that $M_{2}(2 \mathbb{Z})$ is not a weakly completely prime ideal, consider $\left[\begin{array}{ll}3 & 5 \\ 4 & 6\end{array}\right]$ and $\left[\begin{array}{ll}1 & 1 \\ 1 & 1\end{array}\right]$. Now $\left[\begin{array}{ll}3 & 5 \\ 4 & 6\end{array}\right]\left[\begin{array}{ll}1 & 1 \\ 1 & 1\end{array}\right]=\left[\begin{array}{cc}8 & 8 \\ 10 & 10\end{array}\right] \in M_{2}(2 \mathbb{Z})$ but $\left[\begin{array}{ll}3 & 5 \\ 4 & 6\end{array}\right] \notin M_{2}(2 \mathbb{Z})$ and
$\left[\begin{array}{ll}1 & 1 \\ 1 & 1\end{array}\right] \notin M_{2}(2 \mathbb{Z})$. Example 4.12. The zero ideal of the ring $\left[\begin{array}{cc}\mathbb{Z} & 2 \mathbb{Z} \\ 0 & \mathbb{Z}\end{array}\right]$ is a weakly completely prime ideal which is not a completely prime ideal since $\left[\begin{array}{ll}0 & 1 \\ 0 & 0\end{array}\right]\left[\begin{array}{ll}0 & 1 \\ 0 & 0\end{array}\right]=\left[\begin{array}{ll}0 & 0 \\ 0 & 0\end{array}\right]$.

Example 4.13. Let $R=\left\{\left[\begin{array}{ll}a & b \\ 0 & 0\end{array}\right]: a, b \in \mathbb{Z}_{4}, b \in\{0,2\}\right\} . \quad R$ has 2 proper ideals $P_{1}=\left\{\left[\begin{array}{ll}0 & 0 \\ 0 & 0\end{array}\right],\left[\begin{array}{ll}0 & 2 \\ 0 & 0\end{array}\right]\right\}$ and $P_{2}=\left\{\left[\begin{array}{ll}0 & 0 \\ 0 & 0\end{array}\right],\left[\begin{array}{ll}2 & 0 \\ 0 & 0\end{array}\right]\right\} . P_{1}$ is a weakly completely prime ideal which is not a completely prime ideal since $\left[\begin{array}{ll}2 & 0 \\ 0 & 0\end{array}\right]\left[\begin{array}{ll}2 & 0 \\ 0 & 0\end{array}\right]=\left[\begin{array}{ll}0 & 0 \\ 0 & 0\end{array}\right] \in P_{1}$ but $\left[\begin{array}{ll}2 & 0 \\ 0 & 0\end{array}\right] \notin P_{1}$.

Example 4.14. Let $A$ be a ring with $M$ a left $A$-module. Let $Z(A)$ be the set of zero-divisors of $A$ and $(0: M)_{A}=\{a \in A: a M=0\}$ the annihilator of $M$ in $A$. Suppose that $0 \neq Z(A) \subseteq(0: M)_{A}$. Let $[A, M]=\{(a, m): a \in A$ and $m \in M\}$ be 
the ring with componentwise addition and multiplication $(a, m)(b, n)=(a b, a n)$. Now $[0, M]=\{(0, m): m \in M\}$ is an ideal of $[A, M]$. In fact, it is a weakly completely prime ideal, but not a completely prime ideal.

Proposition 4.15. Let $R$ be a ring, $M$ an $R$-R-bimodule, and $I$ a proper ideal of $R$. Then $I \boxplus M$ is a weakly completely prime ideal of $R \boxplus M$ if and only if $I$ is a weakly completely prime ideal of $R$ and for any twin zero $(a, b)$ of $I$ we have $a M=M b=0$.

Proof. Similar as the proof of [1, Theorem 17].

Corollary 4.16. Let $R$ be a reduced ring which is not a prime ring and $M$ an $R-R$ bimodule. Then the unique weakly completely prime ideal which is not a completely prime ideal of $R \boxplus M$ which has the form $I \boxplus M$ where $I$ is an ideal of $R$, is the ideal $0 \boxplus M$.

Proof. Let $I$ be an ideal of $R$ and $J:=I \boxplus M$ such that $J$ is a weakly completely prime ideal which is not a completely prime ideal of $R$. Then $I$ is a weakly completely prime ideal of $R$ which is not a completely prime ideal of $R$ (from [7] we have that $I \boxplus M$ is a completely prime ideal if and only if $I$ is completely prime ideal). From Remark 4.7, $I \subseteq \mathcal{N}_{g}(R)=0$. This means that $J=0 \boxplus M$. The zero ideal $\{0\}$ is a weakly completely prime ideal of $R$. Let $(a, b)$ be a c-twin zero of $\{0\}$. Hence $a b=0$ with $a \neq 0$ and $b \neq 0$. We claim that $a M=M b=0$. Without loss of generality, we may assume $a M \neq 0$. Then, there exists $n \in M$ such that $a n \neq 0$. Now, $(0,0) \neq(0, a n)=(a, 0)(b, n) \in 0 \boxplus M=J$ and neither $(a, 0) \in J$ nor $(b, n) \in J$, a desired contradiction since $J$ is a weakly completely prime ideal of $R \boxplus M$. On the other hand, by Theorem 4.5 and since $J^{2}=0, J$ is not a completely prime ideal of $R \boxplus M$, which completes the proof.

Example 4.17. Let $F$ be a field, and take the free algebra $R:=F<a, b, c$ : $a c^{n} b=b c^{n} a=0, n \in \mathbb{N}>$. $R$ is reduced but not prime. Since $R$ is reduced but not prime it is not completely prime. It now follows from Corollary 4.16 that if $M$ is any $R$ - $R$-bimodule then $0 \boxplus M$ is the unique weakly completely prime ideal which is not a completely prime ideal of the ring $R \boxplus M$.

Remark 4.18. Recall that a ideal $I$ of a ring $R$ is said to have the intersection-offactor-property (IFP) if whenever $a b \in I$ for $a, b \in R$, we have $a R b \subseteq I$.

Proposition 4.19. If $R$ is a ring and $P$ a weakly prime ideal which has IFP then it is weakly completely prime. 
Proof. Let $P$ be weakly prime and $a, b \in R$ such that $0 \neq a b \in P$. Since $P$ has IFP, we have $a R b \subseteq P$. Because $a b \in P$ and $a R b \subseteq P$ we have $0 \neq\langle a\rangle\langle b\rangle \subseteq P$ and $P$ weakly prime gives $a \in P$ or $b \in P$.

Corollary 4.20. If $R$ is a ring and $P$ a weakly prime ideal which is also completely semi-prime then $P$ is weakly completely prime.

Proof. This follows from the fact that a completely semi-prime ideal has IFP.

Theorem 4.21. For a proper ideal $P$ of $R$ the following statements are equivalent:

(1) $P$ is weakly completely prime.

(2) For any subset $B$ of $R$ such that $B \nsubseteq R,(P: B)=\{p \in R: p B \subseteq P\}=$ $P \cup(0: B)$.

(3) For any subset $B$ of such $R$ that $B \nsubseteq R,(P: B)=P$ or $(P: B)=(0: B)$.

Proof. $(1) \Rightarrow(2)$ Let $y \in(P: B)$ where $B \nsubseteq P$. Now $y B \subseteq P$. If $y B \neq 0$ then since $P$ is weakly completely prime it follows from Proposition 4.9 that $y \in P$. If $y B=0$, then $y \in(0: B)$. So $(P: B) \subseteq P \cup(0: B)$. As the reverse containment holds for any ideal $P$, we have equality.

$(2) \Rightarrow(3)$ Suppose $(P: B)=P \cup(0: B)$ where $B$ is a subset of $R$ such that $B \nsubseteq P$. Since $P$ and $(0: B)$ are both subgroups of $R$ it follows from [6] that $(P: B)=P$ or $(P: B)=(0: B)$.

$(3) \Rightarrow(1)$ Let $x, y \in R$ such that $0 \neq x y \in P$. If $y \in P$, then we are done. So suppose $y \in R-P$, then $(P: y) \neq(0: y)$ and from $(3)$, we have $(P: y)=P$. Hence $x \in P$ and we are done.

Corollary 4.22. For a proper ideal $P$ of $R$ the following statements are equivalent:

(1) $P$ is weakly completely prime.

(2) For $x \in R-P,(P: x)=P \cup(0: x)$.

(3) For $x \in R-P,(P: x)=P$ or $(P: x)=(0: x)$.

Proposition 4.23. Any weakly completely prime ideal $P$ in a ring $R$ contains a minimal weakly completely prime ideal.

Proof. Apply Zorn's Lemma to the family of weakly completely prime ideals of $R$ contained in $P$. It suffices to check that, for any chain of weakly completely prime ideals $\left\{P_{i}: i \in I\right\}$ in $P$, the intersection $P^{\prime}=\cap P_{i}$ is weakly completely prime. Let $a$ and $b$ be elements of $R$ such that $0 \neq a b \in P^{\prime}$. If $a \in P^{\prime}$, we are done. So, suppose that $a \notin P^{\prime}$. Then we have $a \notin P_{i}$ for some $i \in I$. Since $0 \neq a b \in P^{\prime} \subseteq$ $P_{i}$ and $P_{i}$ weakly completely prime we have $b \in P_{i}$. Now for any $j \in I$ we have 
$P_{j} \subseteq P_{i}$ or $P_{i} \subseteq P_{j}$. In the first case $a \notin P_{j}$. Since $P_{j}$ is weakly completely prime and $0 \neq a b \in P^{\prime} \subseteq P_{j}$ we have $b \in P_{j}$. In the second case $b \in P_{i} \subseteq P_{j}$. Hence $b \in P^{\prime}$ and therefore $P^{\prime}$ is a weakly completely prime ideal.

Theorem 4.24. Let $R=R_{1} \times R_{2}$ where $R_{1}$ and $R_{2}$ are rings with identities. If $P$ is a weakly completely prime ideal of $R$, then either $P=0$ or $P$ is completely prime.

Proof. Let $R=R_{1} \times R_{2}$ where $R_{1}$ and $R_{2}$ are rings with identities and $P=P_{1} \times P_{2}$ is a weakly completely prime ideal of $R$. We can assume that $P \neq 0$, so there is an element $(a, b)$ of $P$ such that $(a, b) \neq(0,0)$. Now, $(0,0) \neq(a, b)=(a, 1)(1, b) \in P$ and $P$ weakly completely prime gives $(a, 1) \in P$ or $(1, b) \in P$. Suppose $(a, 1) \in P$. Then $0 \times R_{2} \subseteq P$, so $P=P_{1} \times R_{2}$. We show that $P_{1}$ is a completely prime ideal. Let $p q \in P_{1}$, where $p, q \in R_{1}$. Then $(0,0) \neq(p q, 1)=(p, 1)(q, 1) \in P$. Now $P$ weakly completely prime gives $(p, 1) \in P$ or $(q, 1) \in P$. Hence $p \in P_{1}$ or $q \in P_{1}$. So $P_{1}$ is a completely prime ideal of $R_{1}$. The case $(1, b) \in P$ is similar.

Proposition 4.25. Let $A \subseteq P$ be a proper ideals of a ring $R$. Then the following holds:

(i) If $P$ is weakly completely prime, then $P / A$ is weakly completely prime.

(ii) If $A$ and $P / A$ are weakly completely prime, then $P$ is weakly completely prime.

Proof. (i) Let $0 \neq(a+A)(b+A)=(a b+A) \in P / A$ where $a, b \in R$, so $a b \in P$. If $a b=0 \in A$, then $(a+A)(b+A)=0$ a contradiction. Hence $a b \neq 0$ and since $a b \in P$ and $P$ weakly completely prime, we get $a \in P$ or $b \in P$. Hence $(a+A) \in P / A$ or $(b+A) \in P / A$ as required.

(ii) Let $0 \neq a b \in P$ where $a, b \in R$ so that $(a+A)(b+A) \in P / A$. If $a b \in A$, then since $A$ is weakly completely prime, we get $a \in A \subseteq P$ or $b \in A \subseteq P$. If $a b \notin A$, then $0 \neq(a+A)(b+A) \in P / A$. Now, since $P / A$ is weakly completely prime, we get $(a+A) \in P / A$ or $(b+A) \in P / A$. Hence $a \in P$ or $b \in P$ as needed.

Theorem 4.26. Let $P$ and $Q$ be weakly completely prime ideals of a ring $R$ that are not completely prime. Then $P+Q$ is a weakly completely prime ideal of $R$.

Proof. Since $(P+Q) / Q \cong Q /(P \cap Q)$ we get that $(P+Q) / Q$ is weakly completely prime by Proposition 4.25 (i). Now the assertion follows from Proposition 4.25 (ii). 


\section{Rings in which every ideal is weakly completely prime}

We are interested in the structure of rings in which every ideal is weakly completely prime. Note that by definition, a weakly completely prime ideal is a proper ideal of a ring. It is therefore not possible that every ideal of a ring is a weakly completely prime ideal. However, a ring whose zero ideal is completely prime is called a completely prime ring. In this sense, every ring is a weakly completely prime ring since the zero ideal is always weakly completely prime. We may therefore say that every ideal of a ring is weakly completely prime when every proper ideal of the ring is a weakly completely prime ideal. If $R^{2}=0$, then it is evident that every ideal of $R$ is weakly completely prime. In particular, if an ideal $I$ of a ring $R$ is weakly completely prime but not a completely prime ideal, then every ideal of $I$ as a ring is weakly completely prime by Theorem 4.5 .

Proposition 5.1. Every ideal of a ring $R$ is weakly completely prime if and only if for every $x, y \in R$ we have $\langle x y\rangle=\langle x\rangle,\langle x y\rangle=\langle y\rangle$ or $\langle x y\rangle=0$.

Proof. Suppose every ideal of $R$ is a weakly completely prime ideal and let $x, y \in R$. If $\langle x y\rangle \neq R$ and if $\langle x y\rangle=0$ then we done. If $\langle x y\rangle \neq 0$ then $\langle x y\rangle$ is a weakly completely prime ideal. Now, since $0 \neq x y \in\langle x y\rangle$, we have $x \in\langle x y\rangle$ or $y \in\langle x y\rangle$. Hence $\langle x y\rangle=\langle x\rangle$ or $\langle x y\rangle=\langle y\rangle$. If $\langle x y\rangle=R$, then $\langle x\rangle=\langle y\rangle=R$.

Conversely, let $K$ be any proper ideal of $R$ and suppose $0 \neq x y \in K$ for $x, y \in R$. Now we have $\langle x\rangle=\langle x y\rangle \subseteq K$ or $\langle y\rangle=\langle x y\rangle \subseteq K$. Hence $x \in K$ or $y \in K$ and we are done.

Corollary 5.2. Let $R$ be a ring in which every ideal is weakly completely prime. Then for every $a \in R,\langle a\rangle=\left\langle a^{2}\right\rangle$ or $\left\langle a^{2}\right\rangle=0$.

Proof. Let $a \in R$. If $\left\langle a^{2}\right\rangle=R$, then clearly $\langle a\rangle=\left\langle a^{2}\right\rangle$. Suppose $\left\langle a^{2}\right\rangle \neq R$. If $\left\langle a^{2}\right\rangle=0$, then we done, so suppose $\left\langle a^{2}\right\rangle \neq 0$. We have $0 \neq a^{2} \in\left\langle a^{2}\right\rangle$ and $\left\langle a^{2}\right\rangle$ weakly completely prime gives $a \in\left\langle a^{2}\right\rangle$. Hence $\langle a\rangle=\left\langle a^{2}\right\rangle$.

Example 5.3. Let $F$ be a field and $R=F \oplus F \oplus F$. Then for every element $a \in R$, we have $\langle a\rangle=\left\langle a^{2}\right\rangle . I=F \oplus 0 \oplus 0$ is evidently not weakly completely prime, showing that the converse of Corollary 5.2 is false.

Acknowledgements. The author acknowledges useful exchange of ideas with Hisa Tsutsui and Stefan Veldsman on this topic. The author would also like to thank the referee for the valuable suggestions and comments. 


\title{
References
}

[1] D. D. Anderson and E. Smith, Weakly prime ideals, Houston J. Math., 29(4) (2003), 831-840.

[2] A. Badawi, U. Tekir and E. Yetkin, On weakly 2-absorbing primary ideals of commutative rings, J. Korean Math. Soc., 52(1) (2015), 97-111.

[3] P. K. Beiranvand and R. Beyranvand, Almost prime and weakly prime submodules, J. Algebra Appl., 18(7) (2019), 1950129 (14 pp).

[4] G. Birkenmeier, H. Heatherly and E. Lee, Prime ideals and prime radicals in near-rings, Monatsh. Math., 117 (1994), 179-197.

[5] Y. Hirano, E. Poon and H. Tsutsui, On rings in which every ideal is weakly prime, Bull. Korean Math. Soc., 47(5) (2010), 1077-1087.

[6] N. H. McCoy, A note on finite unions of ideals and subgroups, Proc. Amer. Math. Soc., 8 (1957), 633-637.

[7] S. Veldsman, A note on the radicals of idealizations, Southeast Asian Bull. Math., 32 (2008), 545-551.

\author{
Nico Groenewald \\ Department of Mathematics and Applied Mathematics \\ Nelson Mandela University \\ Port Elizabeth \\ South Africa \\ e-mail: nico.groenewald@mandela.ac.za
}

